\title{
15
}

\section{DESIGNER MOSQUITOES?}

\section{Prospects and precautions of genome- edited insects for public health}

\author{
Ramya M. Rajagopalan
}

Some of the most fascinating of the new methods [for controlling insect pests] are those that seek to turn the strength of a species against itself-to use the drive of an insect's life forces to destroy it.

—Rachel Carson, Silent Spring, 1962

Among the many influences theorized across human societies as the animating "life force" giving rise to the splendid diversity of Earth's biomes, perhaps none has been so fetishized by the public and scientific consciousness as the biochemical macromolecule known as deoxyribonucleic acid or DNA: made up of sequences of the nucleotides A, T, G and C, code for genes and genomes throughout the plant, animal and microbial kingdoms. DNA metaphors, like the "book of life" and "the age old language of the living cell," have been critiqued for their over-simplification of life processes (Keller 1995). Nevertheless, the idea of DNA as the animating molecule of life has been fashioned into a ubiquitous icon of heredity, unfairly burdened with the responsibility of an outsized and deterministic influence on the entirety of the intergenerational processes that support life on earth. This "mystique" of DNA as the control centre of all life (Nelkin and Lindee, 2004) is increasingly contested. New research in fields such as epigenetics show that DNA is but one factor among a host of complex biological, social and environmental interactions to which living things are subjected and which shape them at every stage of development, within and across generations. But the chemical and informational simplicity of DNA, and its ubiquity across lifeforms, have become a convenient conceptual platform from which to fantasize about the possibility of manipulating and modifying DNA, and of shaping life into new forms that are subservient to human desires.

The dream of altering or rewriting DNA has been made concrete in the twenty-first century through a set of techniques known as "gene editing" or 


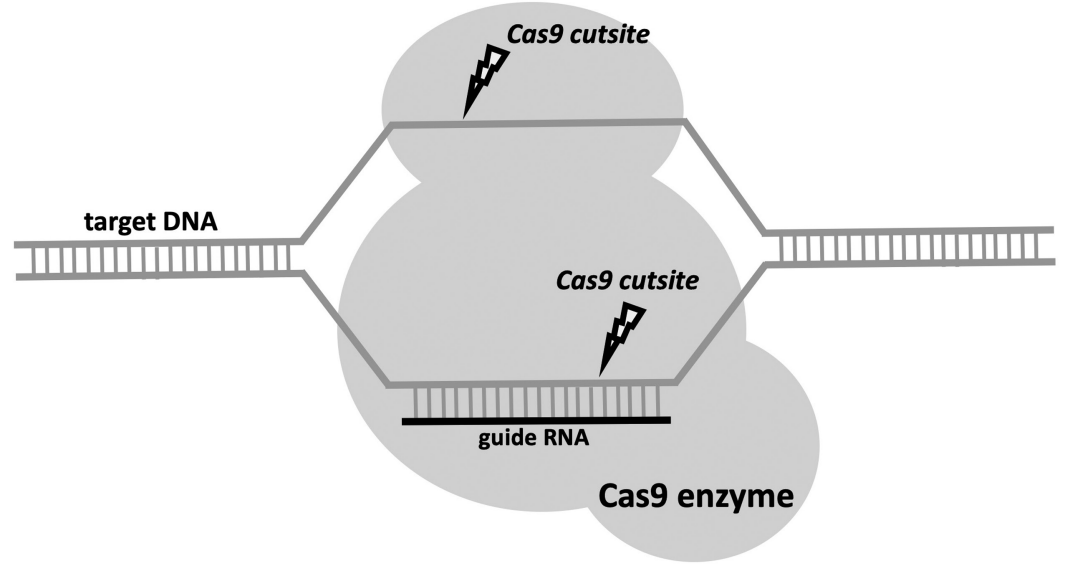

FIGURE 15.1 Schematic of CRISPR-Cas9 mediated gene editing. Source:Adapted from Jinek et al. 2012.

"genome editing," which have transformed scientific research. Gene editing repurposes the bacterial surveillance system known as CRISPR-Cas9, which microbes use as a kind of immune mechanism to identify and defend against invading pathogens like viruses. The catalytic engine of this molecular machine is the enzyme Cas9. Short sequences of ribonucleic acid (RNA) guide Cas9 in locating complementary target sequences in the genome, where Cas9 separates and cuts the two strands of DNA, initiating the rewriting of the target DNA sequence (Figure 15.1). This seemingly simple sequence of steps comprises a powerful molecular technology. Indeed, CRISPR-Cas9-mediated gene editing is just the latest in a long line of genetic engineering tools developed by molecular biologists who have sought to read, write, cut, paste and rewrite DNA to meet human needs.

Since the 1960s, scientists have used genetic engineering to successfully mass-produce vital protein-based drugs like insulin, blight-resistant crops and catalytically active enzymes that form laundry detergent and cheese. Yet many early genetic-modification tools were imprecise, time-consuming and expensive, restricting their possible applications. CRISPR-Cas9 represents a new-if controversial-kind of genetic engineering that can theoretically be used to cut, paste or edit any piece of DNA, in any organism on the planet, relatively easily and cheaply. This technology is potentially world-changing.

Envisioning it as a kind of genetic surgery, enthusiasts see limitless possibilities for gene editing, from curing disease, to improving crops, eliminating insect and fungal pests, and transforming living things into what humans wish them to be. Alongside hopes of transforming health care, agriculture and species conservation, the spectre of CRISPR-Cas9 has also sparked intense debate. This gene-editing technology may allow humans to make irrevocable, 
permanent changes to other species, but its downstream consequences are still poorly understood.

Within global public health, recent efforts have sought to harness gene editing to accelerate the "end of malaria" and other vector-borne diseases. By genetically modifying disease-carrying insects, the aim, borrowing Rachel Carson's framing, is to use the very "life forces" of the vector to destroy itself, or at least to block its ability to transmit the pathogens that have long decimated human populations. As this chapter discusses, the ways in which genetic engineering figures in controlling vector-borne disease illuminates the social, political and economic complexities of utilizing a high-tech approach, challenging our assumptions about the role of human intervention in a delicate global ecosystem. As with any technology, genetic engineering technologies can embody specific political and moral orientations to the problems they seek to address.

\section{The elusive nature of vector control}

Efforts to manipulate the genetic composition of mosquitoes follow on a long history of often frustrated attempts to rid human societies of mosquito-borne disease. For centuries mosquitoes have been viewed as pests, insects whose tiny size belies the throbbing, itchy sting of their blood-sucking bite. But they also serve as unwitting vectors for parasites, like those of the genus Plasmodium, which cause malaria, a debilitating disease that devastates hard-hit communities in South and Southeast Asia, sub-Saharan Africa, and northern South America. Annually, hundreds of thousands of children and adults lose their lives or their livelihoods to malaria (WHO 2020). Among the 112 genera and over 3,500 species of mosquitoes which have been identified, the females of just a handful of species in three genera bite humans to nourish developing eggs, sometimes transmitting disease-causing viruses and parasites. These Anopheles, Aedes and Culex mosquitoes have been the focus of efforts to use genetic approaches for vector control.

In the 1940s, the development of the potent insecticide known as dichlorodiphenyltrichloroethane (DDT) fueled over three decades of vector control and eradication campaigns aimed at alleviating the public health burdens posed by mosquitoes (Stepan 2011). The World Health Organization (WHO) initiated the Global Malaria Eradication Programme in the 1950s in an effort to eliminate malaria in Europe, Asia and the Americas, in part by spraying millions of tonnes of DDT. However, DDT is quite toxic and persistent in the environment, and humanity's growing reliance on it was destroying fragile ecosystems (Carson 1962). Further, mosquitoes rapidly began to develop resistance to DDT and to later pyrethroid-based insecticides that replaced it, even when exposed to previously lethal concentrations.

As eradication attempts faltered and were suspended in the 1960s, mosquito vectors returned and brought with them a resurgence of disease. Some nations and localities temporarily or permanently quashed vector-borne diseases, as the United States did for mosquito-borne malaria, while others struggled with 
rebound malaria. Surviving a bout of malaria could also be protective; when DDT dramatically reduced local mosquito populations and thus the incidence of malaria infections over several years, children no longer encountered the parasite early enough in their development to acquire short-term immunity. Some rebounds were therefore even more deadly than the original epidemics (Cohen et al. 2012).

Into the lively, bloody politics animating failed mosquito eradication campaigns, then, the new DNA editing technologies are being tested as a means of exerting genetic control over insect pests. These high-tech efforts are unfolding in the 2000s in the context of a renewed malaria elimination agenda crafted by the WHO's Global Malaria Programme, in concert with governments and philanthropies. Under their auspices, research groups around the world have pivoted to developing novel chemistries for next-generation insecticides, and more controversially, re-engineering the very DNA of mosquitoes. Twentyfirst-century biotechnology might enhance the arsenal of vector-borne disease control, but not without social, political and ecological ramifications. It also unleashes the power to alter the direction of evolutionary change in target species (Noble et al. 2018). Community members, civil society groups, journalists, scientists, policy-makers, regulators and ethicists, have all raised cautions about deploying genetically edited mosquitoes into the wild.

\section{Gene drives: high-tech mosquito control?}

Several types of genetically modified mosquitoes are currently under development. The British-based company Oxitec has field-tested millions of laboratory-made, transgenic Aedes aegypti mosquitoes in Brazil and the Cayman Islands, in an effort to control dengue, Zika, chikungunya and yellow fever. They have yet to publish epidemiological results from these open field experiments, which could take years. Oxitec engineered male mosquitoes with a gene that kills female offspring; when the modified males are released and mate with wild females, only males survive to adulthood, leading to population collapse. This approach has sparked heated debates about safety, risks and the environmental and health consequences of releasing large numbers of genetically modified mosquitoes, with strong opposition voiced by environmental and civil society groups. Oxitec has also sought to conduct field trials in the United States. In 2016, municipal governments in the Florida Keys held a non-binding referendum asking residents if they would assent to a release of Oxitec's mosquitoes in their neighbourhoods. There was significant opposition to a release (Bloss et al. 2017). Nevertheless in 2020, the US Environmental Protection Agency and mosquito control district boards in the Keys approved the release of Oxitec's mosquitoes, scheduled for 2021.

Another approach known as "gene drive" aims to control malaria-transmitting Anopheles mosquitoes. Gene drives short-circuit the usual patterns of genetic inheritance to ensure that a particular version of a gene is inherited more often 


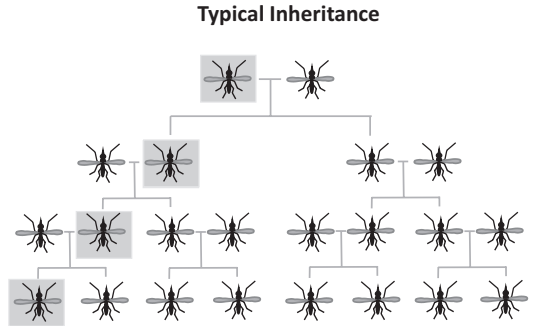

A fraction of the progeny at each generation receive the engineered version of the gene (roughly half, as indicated by the shaded boxes)

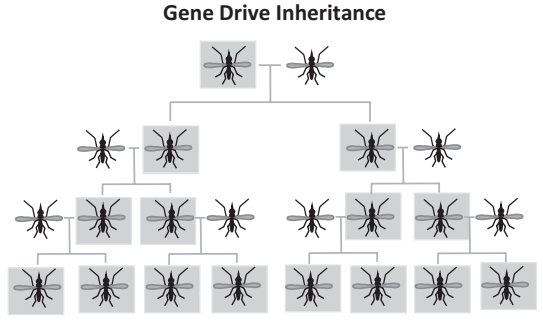

Virtually all of the progeny at each generation receive the engineered version of the gene (as indicated by the shaded boxes)

FIGURE 15.2 Gene drives override typical patterns of inheritance. Source:Adapted from E. Otwell and M. Tefler, in Saey 2015.

than by chance. Most genes are represented twice in animal genomes; each chromosomal copy or "allele" has a roughly equal (50\%) chance of being passed down to a given offspring. A gene drive skews these probabilities in favour of one allele over the other. In a CRISPR-Cas9-based gene drive, gene-editing capabilities are embedded within the target organism itself, permitting the non-engineered allele to be rewritten with the same sequence as the engineered allele (Gantz and Bier 2015a, b). This allows any stretch of engineered DNA chosen by humans to be propagated from generation to generation to both chromosomal copies in virtually all descendants of a mosquito breeding population, as illustrated in Figure 15.2.

Practical applications of gene drives are enormous, but ethically fraught. Gene drives could theoretically be engineered into any target species, to rapidly spread a desired trait to every individual in a wild population. This would give humans the ability to permanently alter any wild species, with just a few genetic tweaks in the lab. Advocates of gene drive, such as some scientists and their funders, are excited by its prospects, framing it as a transformative public health tool that could finally crush the scourge of malaria. They see CRISPR-Cas9 gene-editing technology as a marked improvement over brute-force approaches of blanket insecticide spraying, thereby restricting human-induced changes to only a few species within a diverse geographic area or ecosystem. As philanthropist Bill Gates has written, "The promise of gene editing is that, instead of killing a bunch of mosquitoes indiscriminately, we could eliminate only the dangerous ones in a particular area. That would buy us time to cure all the people there of malaria" (Gates 2019).

Gene drives tantalize with their potential precision, targeting only certain genes in certain species, interrupting the devastating feature of mosquito vectors, which is their role as intermediate hosts for organisms pathogenic to humans. In the public health campaign against malaria, gene drives are being fashioned into "biomedical weapons" (Packard 2007) against two Anopheles vector species, stephensi and gambiae. The Bill and Melinda Gates Foundation and the Tata Trusts 
of India have both committed millions to fund research on gene-drive technology for malaria control, using suppression and replacement gene drives in West Africa, and a replacement gene drive in South Asia, respectively. Both types of gene drive are still in the research and development phase.

\section{Suppression gene drives: eliminate mosquitoes by preventing reproduction}

Target Malaria, a not-for-profit research consortium supported in part by the Gates Foundation, is designing suppression gene drive technology in Anopheles gambiae. The mosquitoes are under development in labs at Imperial College in the UK, as well as in Italy and the United States. Target Malaria is working with stakeholders, regulatory bodies and communities in Burkina Faso, Mali, Uganda and Ghana, to explore the feasibility of field release trials. Their approach exploits an "Achilles' heel" in the mosquito genome creating gene-drive-modified mosquitoes with self-destructive genetic mutations. The mutations would disrupt reproduction, heavily favouring male over female offspring, and rendering any female offspring sterile. If released in the wild, the lab-made male mosquitoes would mate with female mosquitoes and spread the sterilizing mutation to the entire population, resulting over time in a population of mostly males. As with the Oxitec mosquitoes, with fewer and fewer mating partners, the population would eventually crash, effectively wiping it out (Kyrou et al. 2018). Thus, the suppression gene drive could locally eliminate a target species.

\section{Replacement gene drives: eliminate disease by vaccinating the mosquito}

Rather than wiping out target mosquitoes, the second type of gene drive, known as a replacement (or modification) drive, seeks to break their disease transmission capabilities, rendering them harmless. These gene drive mosquitoes are under development at the Tata Institute for Genetics and Society and the University of California at Irvine Malaria Initiative, supported by funding from the Tata Trusts in India and the Gates Foundation, respectively. Engagement efforts are underway to explore the feasibility of field release trials for Anopheles stephensi in India and Anopheles gambiae in São Tomé and Principe. In this approach, engineered mosquitoes would encode small proteins designed to destroy invading Plasmodium parasites, thus "immunizing the mosquito" against the parasite (Aguilera 2020). If released in the wild, modified mosquitoes would mate and spread the parasite-defence genes, halting malaria-causing Plasmodium in their tracks before they ever reach humans. Over several generations, these replacement drives are projected to substitute a disease-spreading mosquito population with one that is incapable of transmitting malaria to humans (Gantz et al. 2015). 


\section{Gene drives: reimagining human-mosquito relations}

While suppression gene drives aim to disrupt mosquito reproduction, replacement gene drives aim to disrupt infectious disease transmission. Importantly, both exploit the natural life-cycle behaviours of mosquitoes, their intricate mating rituals and reproductive movements, to effect human-desired, population-level alterations. Gene drives would harness, in Rachel Carson's words, the very "life forces" of the mosquito to destroy itself or its ability to transmit pathogens to humans. Gene drives therefore represent a new kind of public health tool by conscripting mosquitoes as central actors in the solution to the human health problems they themselves pose.

But suppression and replacement drives carry out their purposes in very different ways, each representing a contrasting vision, and a different set of political and moral orientations to the question of how human-mosquito relationships ought to look going forward. Suppression gene drives posit that a better world is one absent of vector-transmitting mosquitoes, aligning closely with the vision of earlier eradication campaigns. This anthropocentric and arguably reductive view serves to amplify the rhetoric of an age-old war between humans and mosquitoes, their significance to humans and ecosystems defined entirely by the fact that they transmit human disease. This position spotlights the pernicious role of mosquitoes in igniting pandemics that can devastate humans and their governments, economies, and health and welfare systems. It sidelines appreciation of mosquitoes' exquisite biology, the singular evolution of each unique species among a proliferation of thousands, and their remarkable ability to adapt to a variety of ecological niches and climates. Suppression drives reflect a moral orientation to efficiently control - and ideally eliminate - the handful of mosquito species that spread human diseases, so that we may peacefully coexist with the many other harmless mosquito species.

By contrast, replacement gene drives fundamentally reorient our views away from the assumption that mosquitoes are merely subservient to human needs. By rendering mosquitoes harmless, replacement gene drives serve to reimagine mosquitoes as vital parts of ecological systems that are not simply harbingers of disease. If historical campaigns waged against these winged creatures have taught us anything, it is that human efforts to completely wipe out disease-harbouring mosquitoes are likely to fail. Replacement gene drives could foster a new dynamic in human-mosquito relations, encouraging the view that humans and mosquitoes can coexist peacefully and amicably with each other, provided their antagonistic activities are deactivated. Here there is a move beyond the contours of a war metaphor being played out in mosquito eradication campaigns, which rely on a rhetoric of extermination and extinction, to one positioning mosquitoes as vital elements in a humanized world.

\section{Social and ethical concerns around using gene drives for vector control}

At first glance, a replacement gene drive sketches the possibility of a benign outcome dramatically reducing or even eliminating malaria, at least locally, without 
exterminating the mosquito vector. Genetic-control enthusiasts point to what they see as declining efficacies of existing malaria control strategies, such as insecticides and bed nets, due in part to growing resistance among wild mosquito populations. Yet many scientists acknowledge that gene-drive techniques will be insufficient to wipe out malaria, though they may complement existing control strategies in reducing malaria transmission (NASEM 2016). The current director of WHO's Global Malaria Programme, Pedro Alonso, cautions that eradication might be an unrealistic goal even if genetic technologies can be deployed successfully (WHO 2019).

Owing to their ability to irrevocably change species' germ lines, both types of gene drives have become the focus of intense social, political and ethical scrutiny, inciting guarded responses from government leaders, regulators and others tasked with assessing their safety and risk profiles. It is useful to distinguish two, somewhat overlapping sets of concerns around gene drives. The first has to do with social, political and economic dimensions of gene drives as a vector control strategy, raising questions around inequality, wealth, power and poverty within and beyond malaria-endemic regions. The second set of concerns has to do with environmental impacts of gene drives, including ecological risks and orientations around how humans may wish to structure their current and future relationships with mosquitoes and other species in the natural world, of which we are part.

\section{The sociopolitics of gene-drive-modified mosquitoes}

Relations of power, political and economic, typically configure choices and decisions about whether and how a given technology could or should be used. Gene drives are similarly entangled in social and ethical debates that raise questions about the undue influence of power, politics and access. For example, some civil society groups have cautioned against the injection of philanthrocapital into global health concerns, which allows private charities to exert a defining influence on framing priorities and their solutions. Although gene-drive advocates have promised to provide most of the economic resources for developing and deploying gene drives, this strategy represents a substantial financial investment that, some argue, could be used to more effectively reduce malaria in other ways, as discussed below. In addition, research and development on genetically modified mosquitoes is primarily being conducted by labs of the Global North, in the USA and Europe, quite distant from the sites of their intended release in Asia, Africa and Latin America. This raises concerns about how to avoid replicating colonial paternalisms, and how to distribute decision-making authority to those who are most affected by decisions to deploy a gene drive or not. It is important to acknowledge that gene drives are forms of power mediated by individuals and organizations that have likely never had to experience a debilitating life with malaria. Furthermore, not all stakeholders may view the problem as fundamentally one that can be addressed by the techno-logics of genetic solutions. 


\section{Ramya M. Rajagopalan}

Thus, a key consideration for assessing gene-drive-modified mosquitoes is to identify whose interests are served by them. Guidance frameworks for gene-drive development have called for the involvement of diverse stakeholders in transparent, open, equitable and accountable science and decision-making (NASEM 2016). Risks and harms stemming from these technologies are likely to accrue most to those near a release site. Assessing whether communities actually desire gene drives as part of malaria control efforts, and, if so, which design best meets local needs, will require sustained public engagement activities, knowledge sharing and dialogue about potential benefits and harms that are sensitive to local culture, language and religious practices. In many communities, landscapes are culturally significant, and traditions and beliefs about the place of human intervention are built around a long history of careful environmental stewardship. Indigenous communities have valuable local knowledge of disease transmission and ecosystem dynamics, and involving them as active and equal partners in gene-drive design and decision-making could ensure that gene drives do not amplify existing vulnerabilities (Titingfong 2020). Such efforts should ensure that conflicts of interest are minimized by involving a range of stakeholders as partners in decision-making, especially because developers and funders of gene drives are interested parties with respect to decisions about their implementation.

Defining the relevant community could be challenging, as gene-drive-engineered mosquitoes are not likely to obey political boundaries. There are cases of mosquitoes being carried by winds hundreds of kilometres from their point of origin (Huestis et al. 2019) which complicates the use of mosquitoes as public health tools (Biesel and Boete 2013). Areas lying near national or regional borders may be particularly contentious. Some have proposed that every community member be given opportunities to voice opinions, raise concerns and register their free, prior and informed consent, while others suggest that elected or appointed leaders make the final decisions. As gene-drive release areas are proposed, the prevailing political, social and regulatory contexts of each should be accounted for to inform the most suitable approaches for empowering local communities in decision-making about their collective futures.

Finally, some worry that gene drives are reductive and not as cost-effective as other anti-malarial strategies, or that they fail to generate returns commensurate with the expense of creating them. Although CRISPR-Cas9 gene-editing technology is relatively inexpensive, using it to design a gene drive is a very capitalintensive endeavour. Because malaria is concentrated in regions characterized by wide economic disparities, stemming from centuries of exploitive, often violent colonization, affected communities may give first priority to poverty reduction, educational and employment opportunities, and access to adequate health care, over deploying a technology whose efficacy and side effects remain uncertain. Observers of genetic modification approaches have pointed out that alternative means of addressing malaria might have far greater impacts on reducing morbidity and mortality of a mosquito-borne disease, by using large capital injections to shore up health infrastructure, or investing in socioeconomically precarious 
regions in ways that would address inequities in access to housing, education and employment. Such holistic investments would not only help alleviate malaria, a preventable and treatable disease linked to socioeconomic status, but also raise the overall standard of living, ameliorating public health far beyond the quelling of a single disease.

\section{Assessing environmental risks of gene-drive modifications}

Gene drives represent an expensive, high-tech endeavour to address what may be considered fundamentally social problems of vector-borne disease. Today's patterns of mosquito distribution are legacies of military conquest and colonization, stemming from a long history of human encroachment on natural habitats. Major malaria outbreaks have followed human reshaping of natural landscapes which fundamentally altered the distribution and flow of water across the land (Packard 2007). Water is an important feature of malaria transmission because it is in stagnant pools that females lay their eggs and expand their population. In recent times, such landscape modifications often stemmed from redirecting water for power-generating dams or irrigating crops, leading to flooding in some areas and droughts in others.

Given this history, a key challenge for gene drives is to accurately assess their risks to organismal and ecosystem health, and their potential for disrupting relationships between them. The ecological impacts of gene drives are not yet understood. The World Health Organization, the US National Academies, and various national and local regulatory bodies, have all proposed stepwise, phased testing pathways through which gene drives might proceed, beginning with laboratory studies, and moving to confined and open field trials if local communities approve. Iterative rounds of data collection and assessment at each step are critical for evaluating efficacy, safety and environmental, entomological and epidemiological impacts. Could gene drives somehow damage ecosystems or reduce biodiversity? Some have argued that gene-drive-modified organisms may be pervasive and invasive, advising caution (Noble et al. 2018). Others, particularly civil society groups, worry that gene drives may be less precise and targeted than intended, potentially transferring altered DNA sequences to non-target mosquito species, or worse, to other organisms up and down the food chain. Although it still remains unclear how exactly a gene drive would perform in the wild and whether it could successfully eliminate a mosquito species locally, there is much to be learned about how mosquitoes, individually or collectively, interact with complex ecosystems, and what their elimination might mean to the biotic and abiotic environment. Might local elimination of a mosquito species inadvertently lead to local collapse of food webs? Could another mosquito species expand into the territory of the old, occupy its niche, and become a new vector for malaria parasites? What level of knowledge must be attained about knock-on effects and risks to humans, other species, and local ecological webs, before a mosquito gene drive could 
be deemed safe to deploy? Which uncertainties can be tolerated, and which cannot? Are there ways to know if a gene drive is getting out of control, and if so, which measures could be implemented to halt or interrupt the escalating impacts of a renegade drive? Gene-drive researchers are using mathematical modelling to investigate possible impacts on shared environments, and develop mitigation strategies, but few methods currently exist to answer these questions satisfactorily, and better assessment tools must be developed.

And finally, how might gene-drive-modified mosquitoes impair our understanding of and relationships with parasites, malaria and mosquitoes? What might be undesirably lost in our ability to control vector-borne disease through the process of deploying gene drives? For example, gene drives could powerfully shape mosquito evolution, and in turn, shape human susceptibility to mosquitoes. Gene-drive scientists worry about the potential for mosquitoes to evolve resistance to gene drives, just as they did to DDT; such resistances arise readily in the lab. Some wild mosquitoes may find ways to escape the effects of the gene drive, retaining their ability to transmit malaria, and possibly rebounding worse than before. Indeed, any intervention in nature for eliminating or neutralizing a harmful species runs the risk of ultimately aiding its own undoing, even hastening the evolution of new resistances that evade human control. So, while replacement gene drives are designed to convert current vectors into nonvectors, they may also convert these vectors into more virulent varieties (Hayes et al. 2019). Such evolutionary side effects could have long-term implications for future efforts to control malaria. Considered another way, redesigning mosquitoes might also redesign the human experience of mosquitoes, and the malaria they transmit, and not necessarily for the better. Gene drives therefore present planetary scale implications, and the practical and ethical work required to assess and make decisions surrounding them needs to be commensurate with that scale.

\section{Conclusion: the politics of technoscience}

This chapter outlines some of the prospects and precautions of genetic technologies for the control of mosquito-borne diseases. Gene drives are enlivened biotechnologies that aim to tweak mosquito DNA to nudge their biology in directions less pernicious to humans. In so doing, they internalize the problem of vector-borne disease to the mosquito, rather than to the larger web of social, economic, and political inequalities that render such diseases fatal in some bodies and treatable in others. The questions attending gene-drive technologies require us to complicate the assumption that technologies are innocent or somehow apolitical. As theorists of technology remind us, technologies are never ethically or politically neutral. Rather, they are "forms of life" that "restructure our physical and social worlds, and so how we live" (Winner 1983). The technology of gene drive, an altered but living, respiring, mosquito, is doubly a "form of life" (Sandler 2019). Technologies express the ethical and political orientations of their designers, and the futures they wish to implement. The two gene-drive designs of suppression and replacement 
represent starkly different responses to the question of whether disease-carrying mosquito species should exist. The choice of a suppression gene drive expresses a view that disease-carrying mosquitoes are primarily pests and so should be targeted for elimination, whereas a replacement/modification approach sees value in preserving mosquitoes as part of local ecologies, designing a gene drive for compromising only this insect's ability to transmit human disease. In the first scenario, there might not be mosquitoes left to bite humans; in the second, mosquitoes would bite, but not transmit infectious pathogens.

A choice of two mosquito scenarios dovetails into a larger set of dilemmas that loom in the discourse of genetic engineering: to what extent is it acceptable for humanity to manipulate nature to serve its own interests? Is it ethical to edit other species at will, shaping them to have the properties we want or desire, to serve our needs, fancies or whims? Are extreme measures, such as destroying a mosquito species, justified if they help save human lives from a debilitating disease like malaria, which has a disproportionate impact on children made vulnerable by economic inequality, imbalanced power relations and climate change? Would it be unjust not to try to deploy gene drives to eradicate disease vectors?

And yet, gene drives for malaria control can open the door to an ethical slippery slope. Should humans collectively decide it acceptable to genetically alter one species because it serves as a key node in a major health threat, how much easier does it become to justify altering any species, whether viewed as a threat or simply a mild nuisance that may interfere with human agendas and visions? In considering ways to govern decisions about exercising such consequential power, this chapter implores us to nurture humility towards each other and towards the ecosystems of which we are a part.

Genetic technologies dazzle, hypnotically. In so doing, such technologies inflate the illusion of control. But gene drives may not be a saviour. Even if deployed, a gene drive may not be as effective or efficient as anticipated, demanding that we lower our expectations about the human power to "control" mosquitoes or malaria, much less eradicate them. Mosquitoes adroitly and rapidly adapt their relations with us, reacting to our activities, and as with DDT, may resist our efforts to control them. Thoughtful and deliberate interrogations of likely outcomes of our control methods will help us prepare for resistances that are likely to arise.

Suppression and replacement gene drives represent different political and moral orientations to the challenges posed by mosquito vectors. Each gene drive envisions a different future for malaria control and for reorienting mosquitohuman relationships, with competing technopolitics at play in aiming to eliminate or else refashion insect vectors. By seeking opposite avenues for living with and alongside mosquitoes, they may differ in their political, social, ecological and health implications. Gene drives of all stripes threaten to rupture our relationships with mosquitoes, and while this rupture could be beneficial, the journey towards these benefits should also alert us to the dense and complex relations we have with mosquitoes across the political, economic and moral orders of society. 


\section{Bibliography}

Aguilera, M. 2020. Biologists Create New Genetic Systems to Neutralize Gene Drives. UC San Diego News Center, September 18.

Biesel, U. and C. Boete. 2013. The Flying Public Health Tool: Genetically Modified Mosquitoes and Malaria Control. Science as Culture 22(1): 38-60.

Bloss, C. et al. 2017. Public Response to a Proposed Field Trial of Genetically Engineered Mosquitoes in the United States. Journal of the American Medical Association 318(7): 662-664.

Carson, Rachel. 1962. Silent Spring. Houghton-Mifflin.

Cohen, J.M. et al. 2012. Malaria Resurgence: A Systematic Review and Assessment of its Causes. Malaria Journal 11: 122.

Gantz, Valentino M. and Ethan Bier. 2015a. The Mutagenic Chain Reaction: A Method for Converting Heterozygous to Homozygous Mutations. Science 348: 442-444.

Gantz, Valentino M. and Ethan Bier. 2015b. The Dawn of Active Genetics. Bioessays 38: $50-63$.

Gantz, Valentino M. et al. 2015. Highly Efficient Cas9-Mediated Gene Drive for Population Modification of the Malaria Vector Mosquito Anopheles stephensi. PNAS 112(49): E6736-6743.

Gates, B. 2019. Test-Tube Mosquitoes Might Help us Beat Malaria. GatesNotes, April 15, https://www.gatesnotes.com/Health/Test-tube-mosquitoes-might-help-us-beat-m alaria, accessed 11.02.2021.

Hayes, K.R. et al. 2019. Identifying and Detecting Potentially Adverse Ecological Outcomes Associated with the Release of Gene-Drive Modified Organisms. Journal of Responsible Innovation 5(suppl 1): S139-S158.

Huestis, D.L. et al. 2019. Windborne Long-Distance Migration of Malaria Mosquitoes in the Sahel. Nature 574: 404-408.

Jinek, M. et al. 2012. A Programmable Dual-RNA-Guided DNA Endonuclease in Adaptive Bacterial Immunity. Science 337(6096): 816-821.

Keller, E. 1995. Refiguring Life: Metaphors of Twentieth Century Biology. New York: Columbia University Press.

Kyrou, K., A.M. Hammond, R. Galizi, N. Kranjc, A. Burt, A.K. Beaghton, T. Nolan and A. Crisanti. 2018. A CRISPR-Cas9 Gene Drive Targeting Doublesex Causes Complete Population Suppression in Caged Anopheles gambiae Mosquitoes. Nature Biotechnology 36: 1062-1066.

National Academies of Sciences, Engineering, and Medicine (NASEM). 2016. Gene Drives on the Horizon Advancing Science, Navigating Uncertainty, and Aligning Research with Public Values. Washington, DC: National Academies Press. https://doi.org/10 $.17226 / 23405$

Nelkin, D. and S.M. Lindee. 2004. The DNA Mystique. Ann Arbor: Ann Arbor: University of Michigan Press.

Noble, C., B. Adlam, G.M. Church, K.M. Esvelt and M.A. Nowak. 2018. Current CRISPR Gene Drive Systems Are Likely to be Highly Invasive in Wild Populations. eLife 7:e33423.

Packard, Randall. 2007. The Making of a Tropical Disease: A Short History of Malaria. Baltimore: Johns Hopkins University Press.

Patterson, Gordon. 2009. The Mosquito Crusades: A History of the American Anti-Mosquito Movement from the Reed Commission to the First Earth Day. New Brunswick, NJ: Rutgers University Press.

Saey, T.H. 2015. Gene Drives Spread Their Wings. ScienceNews, December 2. 
Sandler, R. 2019. The Ethics of Genetic Engineering and Gene Drives in Conservation. Conservation Biology 34(2): 378-385.

Stepan, Nancy Leys. 2011. Eradication: Ridding the World of Diseases Forever. Ithaca: Cornell University Press.

Titingfong, R.I. 2020. Islands as Laboratories: Indigenous Knowledge and Gene Drives in the Pacific. Human Biology 91(2): 57-67.

Winner, L. 1983. Technologies as Forms of Life. In Epistemology, Methodology and the Social Sciences, eds. Cohen and Wartofsky. Kluwer Academic Publishers.

World Health Organization. 2019. Malaria Eradication, Virtual Press Conference with Pedro Alonso and Michel Tanner, Transcript at https://malariaworld.org/blog/trans cript-virtual-press-conference-pedro-alonso-and-marcel-tanner-malaria-eradicatio n, accessed November 12020.

World Health Organization. 2020. World Malaria Report 2020: 20 years of global progress and challenges. Geneva: World Health Organization. 